\title{
NOTAS
}

1. Cfr. Ricoeur, P.: Tiempo y namación, I: Contiguración del tiempo en el relato historico, Madrid, Cristiandad, 1987, p. 63 .

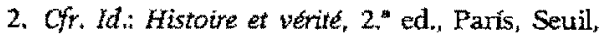
1965 , pp. 96 y 98 .

3. Tal es lo que expone Ricoeur en Etat et violence, conferencia dictada en el Foyer John Knox, de Ginebra, en 1957.

4. Cfr. Zuidema, 5.U. "Original Affirmation and Theological Eschatology in Patl Ricoeur's Thought, especially in his Histore et veritè, en Communication and Confrontation, Kampen (Nederland), J.N. Kok, Lid., pp. 289 ss.

5. Cfr. Ricoewr, P.: "la paradoxe politique", en Esprit, 1957.

6. Fsto lo expresa Ricoeur dia manera muy agu- da en Tiempo y naracion, II: Configuación del tiempo en el relato de ficcion, Madrid, Cristiandad, 1988, pp. 232 ss., donde examina En busca del tiempo perdido, de Marcel Proust.

7. Cfr. Maceiras, M.: "Paul Ricoeur: una ontologia militantew, en Pensamiento, 32 (1976), 131-156.

8. Ricoeur, P.: *Negativite et affirmation ontologiques, en Histoire et vérité.

9. Secretan, Ph.: "La pensée politique de Paul Ricoeur", en Verité et pouvorr, Lausana, l'Âge d'Homme, 1968 , p. 121.

10. $1 d$, p. 128.

11. Ricoeur, P.: "Christianisme prophétiques, en V.AA.: Les chretiens et la politique, Paris, Eds. du Temps Pressent, 1947, p. 13

12. Ibid.

\section{Apuntes sobre la recepción de la fenomenología en España}

\author{
JAVIER LERÍN RIERA \\ Sociedad Española de Fenomenologia
}

El tema que nos ocupa en este breve cstudio es cuestión que presenta sus problemas, pues acometer la tarea que el mismo supone implica, de inicio, tener que delimitar qué comprensión de la fenomenología va a ser la encargada de guiar nuestro trabajo. Con ello no hacemos tanto mención a la interpretación personal que de la doctrina o doctrinas fenomenológicas podamos tener —o pensemos que debería tenerse- como a la misma necesidad de decidir si puede y debe hablarse de la existencia de una o varias fenomenologias. Querer tratar la recepción de la fenomenología en nuestro país conlleva, pues, determinar si vamos a limitar nuestra comprensión de lo que pueda ser entendido como sfenomenología» al pensamiento y la obra de Edmund Husserl -su fundador-o si, por el contrario, vamos a aceptar la existencia de otras fenomenologías. Naturalmente, no podemos negar que el pensamiento de Heidegger y Scheler (por tomar dos ejemplos paradigmáticos) se encuentra de cierta manera emparentado -expresémoslo así- con la filosofía husserliana a pesar de que el propio Husserl afirmara que el camino tomado por el filosofar propio de tales autores fuera contrario a la fenomenología. Por ello, tal vez debiera ser necesario pararse a considerar si ambos pueden o deben ser tenidos por fenomenólogos plenos si enfrentamos la globalidad de su obra con la de Husserl y comparamos entre sí las conclusiones últimas que de cada una de ellas se derivan. Como se comprenderá, la cuestión exigiría una discusión previa que excedería con mucho el 
ánimo que nos guía aunque - de todos modos - no tomar ninguna determinación al respecto equivaldría a quedarnos sin un hilo conductor capaz de marcar el camino a seguir en nuestro estudio. Por otra parte, la necesaria brevedad del mismo parece desaconsejar la idea de acometer su elaboración provistos con una visión excesivamente amplia de lo que con propiedad deba ser entendido como "fenomenología" si es que no queremos contentarnos con tratamientos meramente superficiales sobre lo que ha sido el periplo recorrido por la misma en España. De todos modos, hablar de la recepción de la fenomenología habrá de suponer para nosotros referirse a los avatares sufridos por el tratamiento y la difusión del pensamiento de Husserl en nuestro país aunque, como se comprenderá, ello no debe impedirnos tratar la influencia que ha ejencido la obra de autores como los ahora mencionados Heidegger y Scheler o la atención que ha podido prestarse a otros como Sartre, Hartmann o Merleau-Ponty, ya que obrar de otra forma $-\mathrm{y}$ por más que nuestro personal parecer pudiera incluir la convicción de que la verdadera fenomenología es la husserliana- supondría falsear o sesgar lo que cabe entender bajo el título de urecepción de la fenomenologian.

Sentado todo lo anterior, el siguiente problema afecta a la forma bajo la que desarrollar nuestra exposición. Por razones evidentes, la misma no puede sino tomar la configuración propia de una serie de apuntes sobre aquellos aspectos relevantes por lo que al tema que nos ocupa se refiere; apuntes que, por su parte, deben estructurarse de manera preeminentemente histórica sin tener por ello que convertir el presente estudio en una mera enumeración de autores y obras. Mencionaremos, pues, autores e incluso deberemos hablar de escuelas y tratar de exponer cómo ha sido tomada y comprendida la fenomenolo gía en cada caso. Ahora bien, pretender desarrollar tal planteamiento de forma verdaderamente exhaustiva es algo que nos está vedado por el propio espacio editorial en el que en este instante nos movemos; un estudio verdaderamente completo de la recepción de la fenomenología en España es tema que da holgadamente de sí como para elaborar una obra completa, dado que a la interpretación de la fenomenología efectuada por todos y cada uno de aquellos que en algún instante se han interesado por ella en nuestro país deberian sumarse las diversas comprensiones que del pensamiento de tales autores pudieran tenerse. Junto a ello - y como consecuencia de las mismas causas- nos encontramos con la obligación de tener que presentar al lector una panorámica lo más amplia posible, aun sin pretender que en la misma aparezcan reflejados todos y cada uno de los que en algún instante han prestado atención - para bien o para mal- a la fenomenología ni intentar incluir en la misma un detallado compendio bibliográfico de los estudios sobre ella elaborados por autores españoles, asi como de las traducciones de obras fenomenologicas o de trabajos efectuados en el extranjero. ${ }^{1}$ Así pues, nuestra pretensión es la de elaborar un bosquejo que, aun siendo tal, pueda intentar hacer justicia a lo que ha sido y es la recepción de la fenomenología en España sin que su mismo carácter impida realizar una interpretación de la misma.

$\mathrm{Y}$ si ahora hablamos de interpretaciones no es por capricho sino porque nuestro tema nos fuerza a tener que introducimos en el mismo a partir de la figura de Ortega y Gasset, pensador cuya relación con la fenomenología y su comprensión de la misma no han dejado nunca de ser objeto de cierta polémica. Desde luego, no podemos comenzar si 
no es refiriéndonos a Ortega por el simple hecho de que fue él quien descubrió la fenomenología a la comunidad filosófica española y quien se preocupó de que se diera comienzo a la labor de traducción de la obra de Husserl, hechos éstos que supusieron -entre otras cosas - la puesta en relación directa de Gaos y Morente con el pensamiento fenomenológico. Dejando por el momento a un lado la particular relación mantenida por Ortega con la fenomenología $-y$, en concreto, con la fenomenología de Husserl-cabe llamar la atención sobre el espíritu general que guió la recepción del pensamiento fenomenológico propia de la llamada escuela de Madrid: ${ }^{2}$ tanto Ortega como sus discípulos (estos últimos a partir del primero en un inicio) sufrieron una cierta influencia por parte de la fenomenología, aunque todos ellos tendieron a comprenderla como una forma de pensamiento que debía ser superada a pesar de que pudiera servirles de base para iniciar el recorrido de su propio periplo filosofico. De este modo, podemos considerar que ciertas famosas afirmaciones orteguianas relativas a la fenomenología husserliana expresan de forma acertada lo que parece ser el denominador común presente en la recepción y comprensión de la fenomenología propias de esta primera Escuela de Madrid. En efecto, la fenomenología parece haber sido entendida por dichos autores como una «buena suerte» antes que como una filosofia a la que aferrarse y -consecuentemente- en todos ellos tendía a apuntar una consideración positiva del pensamiento husserliano por lo que hacía a la capacidad del mismo a la hora de servir para diseñar la arquitectónica de un sistema propio; consideración que, sin embargo, se tornaba rápi damente en negativa cuando la fenomenología es tomada en y por sí misma ${ }^{3}$ Para Ortega, formado dentro del neokantismo de la Escuela de Marburgo como discípulo de Cohen y Natorp, la obra husserliana resultó ser una bocanada de aire fresco dentro del panorama filosófico de principios de siglo. Ahora bien (y esta ha sido la interpretación generalmente aceptada de la relación existente entre dicho pensador y la fenomenología), el mismo pareció acabar comprendiendo dicha corriente filosófica como la culminación del racionalismo, como la única forma capaz de otorgar a éste un fundamento sobre el que descansar $\mathrm{y}$-además, $\mathrm{y}$ en cuanto idealismo fenomenológico como el último y máximo exponente de tal tipo de filosofía. Sin embargo, para nuestro autor - si hemos de hacer caso a algunas de sus declaraciones y a las de discípulos suyos como Julián Marías- resultaba necesario tener que pasar por la fenomenología para poder situarse más allá de la misma y adoptar la perspectiva superadora del idealismo que reclamaba para su raciovitalismo. Parece, pues, que la célebre frase de Ortega en la cual afirmaba haber abandonado la fenomenología en el mismo momento de recibirla debería ser tenida en un inicio por cierta (a pesar de ser claramente hiperbólica), así como ser comprendida como una expresión más de la relación que la primera Escuela de Madrid mantuvo con la fenomenología ( $y$, en concreto, con la fenomenología de Husserl). ${ }^{4}$ Ahora bien, tal relación no fue -como ya hemos apuntado antes- $\tan$ negativa como de inicio pudiera parecer, pues incluyó la necesidad de pasar por la fenomenología para poder ir más allá de la misma; consecuentemente, sin la fenomenología resultaría imposible la continuación de la reflexión filosófica. Por ello, si bien los miembros de tal escuela parecieron recibir la fenomenología para abandonarla acto seguido, también debe repararse en el hecho de que sin ellos -con Ortega al frente- no hubiera sido conocida en España, ya que el deseo de tales autores 
de hacer comprender sus propios desarrollos filosóficos necesitaba que el público académico pudiera conocer de una forma directa los textos estrictamente fenomenológicos donde, desde su punto de vista, se había consumado una tradición. $\mathrm{Y}$ hablar de textos fenomenológi$\cos$ suponía referirse a Investigaciones Logicas y al primer tomo de Ideas, obras cuya traducción fue realizada por Morente y Gaos. 5 Verdaderamente, la primigenia influencia de la Escuela de Madrid en la recepción española de la fenomenología llevó a configurar la convicción - vigente aún de forma virulenta entre muchos de los actuales seguidores de Ortega- de que el quid del pensamiento husserliano $(y$, por ende, de la fenomenologia en sí) se encuentra encerrado en ambas obras, lo cual ha supuesto durante mucho tiempo una reificación negativa de la comprensión de la fenomenología que se contenta con referirse al «idealismo» de la segunda de tales obras y entender todo desarrollo posterior del pensamiento de Husserl a partir de ella. No queremos con ello afirmar - obviamente- que los miembros de dicha escuela desconocieran la producción posterior de Husserl, sino que su contacto original con la fenomenologia (el contacto original de Ortega, en concreto) tuwo como sucesivos protagonistas a tales obras y que la idea que de ella se hicieron los mismos maduró a través del inicial estudio de ambas e influyó sobre el resto de la comunidad filosófica hispanoparlante. Prueba de que, de todos modos, su conocimiento de la obra de Husserl fue más allá de todo ello la tenemos en la primera traducción de las Meditaciones Cartesianas, realizada por Gaos, y en el siempre cercano seguimiento de la actividad académica y editorial de Husserl realizado por Ortega; seguimiento que podría quedar reflejado en su sorpresa —expresada en sus Apuntes sobre el pensamiento - ante las conferencias de Praga de 1935, a partir de las cuales Husserl construiría buena parte del armazón de Crisis, su último trabajo. ${ }^{5}$

El problema que suscita la comprensión orteguiana de la fenomenología se mueve en diversos frentes. Por una parte, la filosofía de Ortega ha sido muchas veces injustamente atacada al afimarse de la misma que no era un pensamiento original, lo cual --desde nuestro punto de vista- no puede ser mantenido. Que ciertas posturas alcanzadas por Ortega coincidieran en mayor o menor medida (y aqui volvemos a encontramos con el problema de la interpretación, que ahora nos vemos obligados a hacer a un lado) con las de un primer Heidegger que comenzaba a distanciarse de la fenomenología estrictamente husserliana para nada prueba que las mismas fueran una parafrasis de lo mantenido por este último autor. La reflexión madura de Ortega partía, al igual que la de Heidegger, de una determinada etapa del desarrollo de la fenomenología husserliana y se centraba - en cierta medida - sobre algunos temas comunes. Por ello, la aparición de determinadas concomitancias o resonancias no puede -o no habría debido- dar lugar a vanas reticencias mientras que, por otra parte, un serio análisis de la obra de Ortega bastaría para demostrar cómo ciertas opiniones que habrian podido caer bajo tan injusta sospecha aparecian ya en sus Meditaciones del Quijote, anteriores en más de una década a la publicación de Ser y tiempo. Lejos de solucionar plenamente la cuestión, toda esta serie de afirmaciones nos reenvía a la génesis del problema suscitado por la recepción y comprensión orteguianas de la fenomenología al poder llevar a la conclusión de que la insatisfacción de Ortega con respecto a la filosofia de Husserl debe ser comprendida como una actitud mantenida de forma continuada y sin aparentes matices. Sin embargo, acabamos de hablar de la sor- 
presa que en Ortega parecieron causar las conferencias de Praga (cuyo texto conoció cinco años después de su elaboración) e, igualmente, acabamos de mantener que la reflexión orteguiana parte de un determinado instante dentro del desarrollo de la fenomenología husserliana. Ambos datos señalan --según nuestro parecer- la solución a esta cuestión, porque si bien es cierto que Ortega vio en la fenomenología del primer tomo de Ideas el colofón a un tipo de filosofía que había que superar -aunque fuera asumiéndola- también lo es que en 1929 Husserl consideraba igualmente a dicha obra como superada ${ }^{7}$ y que su pensamiento, siempre en continua y permanente cvolución, comenzaba a mostrar aspectos hasta entonces insospechados. Ortega, quien siguió siempre la producción husserliana, pudo ir interpretando las diversas obras de Husserl a la luz de la comprensión estereotipada que de la fenomenología se había formado en su momento pero el agiro" del "último" Husserl demostraba cómo dicho autor había desarrollado su pensamiento de forma concomitante (en ciertos aspectos, algunos de ellos nucleares) a la del propio Ortega hasta un punto tal que éste llegó a atribuir aquel texto de 1935 a Fink, quien comenzaba a decantarse hacia Heidegger a pesar de mantener incólume su lealtad hacia el fundador de la fenomenología. ${ }^{*} \mathrm{Si}$ a todo cllo unimos el hecho de que en 1934 Ortega habia viajado a Friburgo por propia iniciativa con el fin de visitar a Husserl, deberíamos concluir que su relación con la fenomenología no puede ser comprendida como la historia de una primera admiración a la que después seguiria un rechazo total e inamovible, sino como algo bastante más fluido y mucho menos roturdo. De hecho, creemos que releer la obra de Ortega provistos con la mentalidad que tales datos pueden proporcionamos debería otorgar toda una scrie de conclusiones que, desde luego, tienden a chocar con la comprensión de la recepción y vigencia del pensamiento fenomenológico en la filosofía de dicho autor que él mismo ayudó a propiciar y que sus discípulos y muchos de sus seguidores - pasados y presentes- aún fomentan. Tal tipo de lectura puede llevar, pues, a la consideración de que en Ortega hubo siempre más fenomenologia de la que parecía traslucir, lo cual - a su vez- exigiría prestar una mayor atención a aquellos de sus escritos más claramente vinculados con su recepción de la misma de cara a efectuar una evaluación general de su pensamiento. De esta manera, trabajos como las antes citadas Meditaciones del Quijote pueden recibir una nueva interpretación, otros como Sobre el concepto de sensación ser tenidos como más decisivos de lo que generalmente se ha admitido y llegar a descubrir una pervivencia de la fenomenología y su método en estudios como Ensimismamiento y alteración, por citar algunos ejemplos. ${ }^{10}$

Dcjando ya de lado a Ortega, cabe resaltar que esta primera recepción de la fenomenología en España no supuso tan sólo la introducción y el inicio del conocimiento en nuestro país de la obra de Husserl. Como resulta comprensible, la fulgurante aparición de Heidegger en el panorama filosófico como pensador con una verdadera entidad propia, acaecida gracias a la aparición de su Ser y tiempo en 1927, no pasó inadvertida en España y ya antes de la traducción de dicha obra - debida también a Gaos - tal autor polarizó buenas dosis de atención (en detrimento -incluso- del propio Husserl, como igualmente sucedia en otras latitudes). La filosofía de Zubiri -por poner un ejemplo- tomó como basamento (al igual que la de muchos de los miembros de la Escuela de Madrid) ${ }^{11}$ la fenomenología, encontrando en Heidegger (con quien estudio en Fribur- 
go) un punto de partida sobre el que después volvería críticamente en debate preferentemente construido a partir del problema supuesto por el objetivismo y el realismo. De todos modos, no puede considerarse que el contacto de Zubiri con Heidegger le llevara a olvidar la fuente originaria de la fenomenología, pues la filosofia husserliana fue también - y de forma necesaria- objeto de su interés (de lo cual puede uno cerciorarse con simplemente ojear su Naturaleza, Historia, Dios). Por su parte, su análisis de la Inteligencia sentiente contenido en su trilogía Inteligencia sentiente, Inteligencia y logos e Inteligencia y razón guarda en su transfondo una clara influencia fenomenológica (aunque sólo sea por lo que al carácter general de la misma y al modo general de proceder se refiere), ${ }^{12}$ lo cual impide considerar a dicho autor como alguien separado de la fenomenología por más que se apele al ascendente que en su pensamiento pudieron tener la filosofia netamente aristotelica y la propiamente escolástica.

Pero esta primera 'irrupción' -llamémosla así- de la fenomenología tampoco acaba con Heidegger, pues la tarea de traducción de la producción fenomenológica inicialmente alentada por Ortega se concretó en la aparición en español de textos de autores como Pfänder (quien ya en vida de Husserl presentó una versión propia del método fenomenológico, originalmente debido a aquél). ${ }^{13}$ En este apartado resulta relevante - junto a Gaos-la figura de Morente, a quien en un inicio atrajo vivamente la filosofía de Scheler sobre la de los demás pensadores vinculados en mayor o menor grado a la fenomenología (atracción a la que se unía su simpatía por Bergson). Creemos que a la luz de sus trabajos puede afirmarse que Morente, quien como el resto de los pensadores de la Escuela de Madrid había recibido la fenomenología a través de Ortega, comprendía en buena parte a ésta como método y que, justamente, consideraba al método fenomenológico como una optima berramienta de trabajo aunque - desde nuestro punto de vista- lo relevante de la relación mantenida por dicho autor con la fenomenologia sea su posterior interés (tal vez continuación de aquella "sorpresa" orteguiana a la que antes hacíamos referencia) con respecto a los últimos desarrollos del pensamiento husserliano, en los que detectaba un paralelismo con el raciovitalismo. Por su parte, Gaos tiende a parecer sobre todo un historiador de la filosofía con un largo peregrinar a sus espaldas, buen conocedor de los diversos sistemas y doctrinas y -por ellode la fenomenologia. Naturalmente ( $\mathrm{y}$ al igual que ocurría con Morente), su ocupación de traductor suponía el contacto y el conocimiento de los grandes y pequeños sistemas que por aquellos años perfilaban el panorama filosóficó conocimiento al que se unía - como sucede con todos los autores hasta ahora tratados y por tratar - una sólida formación escolar. La figura de Gaos es, junto a la de Ortega, la del gran divulgador de la fenomenología en todo el ámbito hispanoparlante a través de sus ya mencionadas traducciones, sus obras y su labor docente, realizada principalmente en Hispanoamérica (donde se exilió tras nuestra poco grata de recordar contienda civil). Ahora bien, que Gaos deba ser rectamente tenido por divulgador de la fenomenología no supone su adhesión incondicional o acrítica a la misma. Si tomamos como punto de referencia su Filosofia contemporánea, podremos comprobar cómo -en efecto- dicho autor era perfecto conocedor de las más diversas corrientes filosóficas y cómo era capaz de sacar a la luz las ocultas relaciones que las vinculaban entre sí (aunque fuera como resultado de reacciones $u$ oposiciones), ofreciendo así 
una comprensión global del panorama de la época por lo que al campo del pensamiento se refería; e, igualmente, podremos cerciorarnos de que Gaos conocía y comprendía de forma altamente rigurosa todos los desarrollos de la fenomenología (desde Husserl a Hartmarn, pasando por Scheler y Heidegger), siendo capaz de exponerlos de forma clara y brillante sin necesidad de perder un espiritu crítico. ${ }^{14}$ Ahora bien, Gaos no se agota como historiador de la filosofía y expositor de sistemas sino que, junto a tal faceta, nos encontramos con aquel peregrinar al que antes hemos hecho mención. Comenzando por el raciovitalismo de Ortega, Gaos se movió de una filosofía a otra a partir de una postura teñida de marxismo (lo cual le supuso pasar por Husserl y Scheler) hasta alcanzar un pensamiento propio: el personismo. Ahora bien, por lo que a nosotros nos interesa cabe resaltar su preocupación antropológica y su afirmación de que los dos rasgos característicos del hombre son la mano y el tiempo. ${ }^{15}$ En el primero de ellos - la mano - encontramos la impronta mar. xista que lleva a considerar al hombre -acertadamente, según nuestro parecer- como homo faber, mientras que la apelación al segundo -el tiempo- denota un ascendente claramente fenomenológico que señala tanto a Heidegger (el punto de referencia más inmediato para el profano) como a Husserl. ${ }^{16}$

Mientras se mantuvo vivo el magisterio directo de los miembros de la Escuela de Madrid ${ }^{17}$ la fenomenología pudo ir siendo conocida en nuestro país, aunque su divulgación hubo de suponer su necesaria vulgarización con el paso del tiempo; vulgarización que -como antes hemos mencionadoacababa viendo el non plus ultra de la misma en Investigaciones Lógicas y el primer tomo de Ideas, to cual conllevó -y aún conlleva - un desinterés por la misma al considerarla como el canto del cisne de un idealismo trasnochado o como una extraña reedición de un caduco platonismo. No puede hablarse de una persecución de la fenomenología sino más bien de un olvido de la misma. En todo caso, la figura señera de Heidegger supo -0 , al menos, a nosotros nos lo parece- capear el temporal con entereza, pues sus últimos desarrollos pudieron ser vistos como algo ya totalmente ajeno a su primitiva relación con Husserl. De todos modos, la fenomenología fue dada por muerta más de tuna vez a medida que nuevas filosofías hacían su entrada en España. El empuje del existencialismo de Sartre -a pesar de su vinculación con Husserl- fue generalmente tomado como algo que señalaba el necesario abandono de la fenomenologia, tachada a veces $\sin$ ambages de reaccionaria al haber encontrado eco en algunos pensadores de orientación neoescolástica, lo cual tendía a convertirla en una filosofía no molesta (o no demasiado molesta) para el statu quo. Desde luego, la significación -o significaciones- filosóficas del 68 francés y el auge del marxismo en las facultades (un marxismo a veces resultado de la moda y, por ello, bastante dispuesto a disolverse en algunos casos cuando los imperativos de la moda han cambiado) no parecía propiciar la existencia de un caldo de cultivo óptimo para la fenomenología que, en todo caso, pasaba a engrosar la lista de grandes sistemas archivados. Sin embargo, la misma -aunque cada vez peor conocida- logró captar la atención de ciertos círculos académicos; atención creciente a medida que los inéditos de Husserl iban viendo la luz y en la medida en que la labor de traducción de sus obras era mantenida en Hispanoamérica. De este modo, en un panorama de inicio no demasiado propio para ello aparecieron lo que podemos con toda 
propiedad considerar como nuevas escuelas en la recepción de la fenomenología. Por una parte, la Universidad Complutense de Madrid vio aglutinarse en torno a dos de sus profesores - Sergio Rábade y Millán Puelles- a un grupo de alumnos que, como en los buenos tiempos de la Escuela de Madrid, estaban lo suficientemente interesados en aquella filosofía como para dedicarle el esfuerzo que la elaboración de una tesis de licenciatura o doctorado puede exigir. La recepción de la fenomenología propia de S. Rábade podría ser calificada como poseedora de un claro carácter académico y orientada - en lo fundamental - a lo gnoseológico. ${ }^{18}$ De hecho, las preocupaciones de Rábade y su escuela (pues creemos que de escuela puede hablarse) eran eminentemente críticas y venian principalmente marcadas a partir del estudio de Kant, generando un "redescubrimiento" de la fenomenología que iría reflejando la amplitud de miras propia de la misma. Con Rábade trabajaron -entre otros muchos- J.L. Arce (la primera persona a quien oímos hablar de fenomenología y gracias a la cual hoy se mantiene viva la divulgación de la misma en la Universidad de Barcelona merced a las actividades de su cátedra) y J. San Martín (catedrático de la UNED y plenamente dedicado al "proselitismo" fenomenológico -digámoslo así- a través de la docencia y la publicación). ${ }^{19}$ La escuela encabezada por Millán Puelles efectuaria -hablando a grandes rasgos - una recuperación e interpretación de la fenomenología basada en un apoyo escolástico y, en áltimo término, confesional o -cuando menos- teista. ${ }^{20}$ De entre los múltiples discípulos que ha tenido resulta obligado destacar a $M$. GarcíaBaro, profesor de la Universidad Complutense, gran conocedor de Husserl, recuperador de la original labor de traducción iniciada por Morente y Gaos ${ }^{21}$ y defensor de una fenomenología en la que to teleológico y lo monadológico permiten la introducción de lo teístico. Junto a el, puede mencionarse también a I. Gómez Romero, situado en perspectiva semejante y autor de un libro en el que divulga la misma. ${ }^{22}$ Por su parte, merece también importante mención la que puede Ilamarse Escuela de Valencia, con el profesor Montero Moliner al frente, dedicado desde hace años al estudio de la fenomenología y curya idea de retornar a la misma supone una comprensión samplia» del pensamiento husserliano en la que no deja de ser patente -entre otras - una cierta influencia de la que podría ser llamada Escuela Lingüística valenciana, debiéndose hacer también referencia a Jesús Conill de quien -entre otros rasgos- podemos mencionar su reivindicación de Zubiri como fenomenologo. ${ }^{23}$ Haciendo un breve recorrido por otras universidades españolas, deberíamos mencionar a César Moreno (profesor de la Universidad de Sevilla y autor del -para nosotros- más completo estudio dedicado al tema de la intersubjetividad en Husserl elaborado en España y traductor de algunos textos del mismo), ${ }^{24}$ al grupo de profesores de la Universidad de Santiago de Compostela (Nel Rodríguez, M. Luz Pintos, M. Riobóo) que ven en la fenomenología el modo de hacer filosofía a seguir e -incluso- a la Escuela de Oviedo, encabezada por Gustavo Bueno y que - pese al materialismo que la caracteriza- no deja de prestar atención a la fenomenologia. ${ }^{25}$ Igualmente, debemos referirnos a Jacobo Muñoz (Universidad Complutense) por su traducción de Crisis y a Gómez Heras (de la Universidad de Salamanca) quien -desde su interés por Bloch- no ha tenido reparo en reconocer la importancia del uúltimo» Iusserl, dedicándose a la claboración de una obra centrada en los aspectos éticos derivados de lo manteni- 
do por el mismo. ${ }^{26} \mathrm{Y}$, por último $(\mathrm{y}$ aun sabiendo que muchos otros han quedado injustamente sin mención) debemos referirnos a la Sociedad Española de Fenomenología (SEFE), formada a partir de la celebración del Primer Congreso Mundial de Fenomenologia (que tuvo lugar en 1988 en Santiago de Compostcla), donde los promotores iniciales de la misma (Montero, Nel Rodriguez, San Martín...) pudieron comprobar cómo había renacido el interés por la fenomenología en nuestro pais hasta el punto de poder pensar en dar nacimiento -hecho ocurrido en octubre de 1989 - a la SEFE (cuyo presidente honorario es el ahora mencionado F. Montero y su presidente el igualmente nombrado $\mathrm{J}$. San Martín), fuero de trabajo abierto a cualquier comprensión de la fenomenología y de la que forman parte buen número de los ahora mencionados junto a otros tantos (licenciados, doctorandos, profesores universitarios y de enseñanza media, etc.) cautivados por la fenomenología. La actuación y el talante de la misma es lo suficientemente amplio como para contener en su seno a husserlianos netos de una u otra orienta- ción, interesados en Merleau-Ponty, en la hermenéutica (como J. Osés, de la Universidad Pública de Navarra) o en la fenomenología linguística de Austin, Searle e -incluso- en Habermas (como V. Martínez, de la recién creada Universidad de Castellón). Los trabajos de la Sociedad comenzaron con la reivindicación de Ortega como fenomenólogo, continuaron con el tema de la Lebenswelt y ahora - siguiendo con esta temática - apuntan hacia la interculturalización. Su serio ritmo de trabajo incluye la celebración de un Seminario Permanente a lo largo del curso académico y su culminación en una anual Semana Española de Fenomenología.

Como se ve (y pese a un olvido que pudo llevarla hasta la desaparición), la fenomenología es todo menos una filosofía muerta en España; en concreto, resulta capaz de despertar-intereses y aglutinar esfuerzos investigadores, docentes $y$ divulgativos y gracias al trabajo de la SEFE - puede pensarse que su conocimiento será aún mayor cuando la tarea de traducción de los textos de Husserl aún inéditos en español comience a salir a la luz. ${ }^{27}$

\section{NOTAS}

1. Una buena información de este tipo puede encontrarse en J.A. Arias e l. Gómez Romero, $M a$. teriales para una historia de la fenomenologia en España, publicado en la revista Fragua (Madrid), 23. 24 (1983), 14-39. Por nuestra parte (y dentro de las limitaciones antes señaladas), intentaremos cubrir de la mejor manera posible el período comprendido entre la elaburación de este estudio y el momento presente.

2. Mencionar a la Escuela de Madrid ha supuesto siempre -por tradición- referise al grupo formado por Ortega y sus discfpulos, tal y como nos. otros mismos acabamos de hacer. De todos modos, tratando de la recepción de la fenomenologia ca bría la posibilidad de precisar y hablar de una Primera Escuela de Madrid para hacer expresa mención a dicho grupo de pensadores, pudjendo así diferenciarlo de las que podríamos llamar Nuevas Escuelas de Madrid, cuyos iniciadores habrían sido los profesores de la Universidad Complutense S. Rábade Romeo y A. Millán Puelles.

3. En palabras de Ortega: «Su valor inestimable [el de la fenomenología] está en la "fina estructura" de tejidos camosos que puede ofrecer a la arquitectura de un sistema. Por eso, la fenomenología no fue para nosotros una filosofía: fue ... una buena suertew (Prologo para alemones, en Obras Completas, vol. VIII, p. 42).

4. La afirmación de Ortega, que hemos vertido de forma prácticamonte literal en el texto, es en concreto la sigujente: «De esta manera abandone la Fenomenologia en el momento mismo de recibirlas (La idea del principio en Leibniz, en Obras Complem tos, vol. VII, p. 273). Por lo que a la interpretación de la relación existente entre Ortega y la fenomenologia efectuada por Julián Marias se refiere, puede valer el siguiente fragmento de su conocidisima Historia de la filosofia: "La fenomenología, al pen- 
sarla a fondo, nos descubre su última raiz errónea [es decir; su incapacidad de superar los presupuestos idealistas] y nos deja fuera de ella, rnás allá de ella.... (O. C., 30." ed., Madrid, Revista de Occidente, 1978, p. 436$\rangle$.

5. En concreto, la traducción de Investigaciones Lógicas fue llevada a cabo por ambos autones y publicada por primera vez por Revista de Occidente en Madrid el ano 1929, mientras que la del primer tomo de Ideas fue obra individual de Gaos, siendo publicada por primera vez en 1949 por FCE en México. Ambas obras han sido reedicadas varias veces con el ticmpo; la primera por su editorial original y, úlimamente, por Alianza Editorial, mientras que la segunda continúa siendo editada por el Fondo de Cultura.

6. La traducción de Meditaciones Cantesianos realizada por Gaos fue publicada en 1942 por El Colegio de México con el añadido de un prólogo compuesto por dicho autor. Dicha edición contaba con la peculiaridad de presentar tan sólo las cuatro primeras Meditaciones de las cinco que confoman dicha obra de Husserl. Este hecho parece deberse a que el propio Gaos extravió el texto de la quinta $M e-$ ditación, tal y como refiere A. Zirión en la presentación de su traducción de las llamadas Conferencias de Parts (México, UNAM, 1988, p. V, nota 1). A la incompleta traducción de Gaos siguió la ya completa del argentino Mario A. Presas (que antade las observaciones criticas de Ingarden contenidas en el primer tomo de Husseriana, en el que se encuentra editada dicha obra junto a las ahora referidas conferencias en las que tuvo su origen), originalmente publicada el año 1979 por Ediciones Paulinas en Madrid y luego vielta a editar el año 1986 en la misma ciudad por la editorial Tecnos. Ese mismo año el Fondo de Cultura reeditaria la traducción de Gaos, anadiêndole una traducción de la antes ausente quinta Meditación realizada por M. Garcia-Barb, profesor de la Uniwersidad Complutense, discipulo de Millán Puelles y - para nosotros- uno de los mejores traductores de la obra de Husserl (si no el mejor) junto a las figuras señeras de Gacs y Morente. Por lo que a Crisis respecta, hoy contamos ya con una traducción de la misma, editada por Catedra en Madrid el año 1991 y realizada por Jacobo Muñoz y

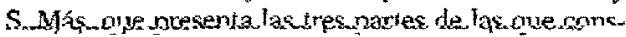
ta en sí dicha obra más los textos de conferencias que aparecen en el sexto volumen de Husseriana aunque por cuestiones editoriales (dolorosamente comprensibles hoy en dia, por desgracia) no contio ne los diversos apéndices reunidos por Biemel. Hasta la tan tardín aparición de esta traducción las únicas traducciones al español disponibles $-y$, aun así, dificilmente- fueron las hispanoamericanas, muchas de ellas incompletas, algunas realizadas en reducidisima tirada y para su exclusiva distribución dentro de algunas universidades, y otras piratas. De entre todas ellas, la más utilizada y accesible últinamente -a pesar de sus imperfecciones- ha sido la efectuada por H. Steinberg y publicada en México el año 1984 por Folios Ediciones.

7. A este respecto, véase la p. XXXIII de la introducción de Iso Kern a Husserliana, vol. XIII, Zur Phanomenologie der Intersubjektivität $I$, donde dicho autor cita una carta de Husserl a $G$. Misch de dicho ano en la que aquél se refiere al primer tomo de Ideas calificandolo de desanollo inicial e imperfecto de la fenomenología.

8. La opinión de Ortega con respecto a las conferencias de Praga del año 1935 en las que Crisis tuvo su origen $y$ a la que venimos refiriêndonos con insistencia se encuentra en el anejo Razón vital e Idealismo (correspondiente a Apuntes sobre el pensamiento), donde Ortega - tras haber atacado el idealismo que siempre había detectado en Husserl como algo insuperable desde dentro de la propia fenomenología- añade a renglón seguido de la última nota: "Al tiempo de corregir estas pruebas me entero, por un azar, de que en 1935 Husserl dio una conferencia en Praga [...] Para mí ha sido sumamente satisfactorio este brinco de la doctrina fenomenológica...) (O, $C$, Madrid, Revista de Oocidente, col. El Arquero, 1959, p. 50). A continuación de lo ahora transcrito, la sorpresa de Ortega le lleva (tal y como acabamos de señalar en el texto) a apuntar la posibilidad de que dicho trabajo hubiera sido realmente elaborado por Fink aunque su autoría le fuera achacada a Husserl.

9. De dicha visita nos quedan dos testimonios de primera mano: el del propio Ortega y el de Husserl, cada cual más significativo. En La idea del principio en Leibniz (ed. cit. p. 273, nota 2) Ortega se reliere a la misma hablando del eadmirable Husserl quien, debido sa [su] edad y [sus] enfermedadesn, no pudo dedicarle todo el tiempo que hubiera sido de desear. aunque le remitió a Fink, «discípulo suyo, ejemplarmente dotadom. Por su parte. Husser] da cuenta de tal visita con las siguientes palabras en carta dirigida a Ingarden: «Esta semana hernos recibido la muy interesante visita filosofica de Ortega y Gasset, quien nos ha dejado vivamente impresionados. Durante todos los días ha mantenido largas y profundas conversacjones conmigo y con Fink. De hecho, no es sólo [x.] el educador de la nueva España, sino tam-

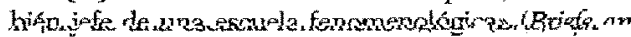
Ingarden, en phatomenologica, wol. 25, La Haya, M. Nijhoff, 1968 , p. 90 , carta del 26 de noviembre de 1934). Naturalmente, sobre esta úlima afirmación de Husserl con respecto a Ortega cabria preguntarse si la misma procede única y exclusivamente del propio Husserl o si, por el contrario, fue el mismo Ortega quien le dio pie a realizarla.

10. De hecho, dicha tarca fue ya empezada por la Sociedad Espantola de Fenomenología (SEFE) -a la que con posterioridad nos referiremos en el textodurante las sesiones de su primer Seminario Pemanente (mantenidas a lo largo del cunso académico 89/90) y las de la Primera Semana de Fenomenolo- 
gra (celehrada en Madrid en Septiembre de 1990), dexicadas a la reivindicación del carácter fenomeno. lógico de la filosofia orteguiana y a las que acudieron miembros de la Fundación Ortega y conocedo. res de su obra como-entre otros- P. Cerezo y N. Orringer. Es de esperar que las conclusiones de todo el trabajo entonces realizado vean pronto la luz, lo cual puede abrir un fructífero debate en tomo a la fenomenologia y su recepción y pervivencia en Ortega y sus discípulos. Un ejemplo de lo que supone comprender a Ortega como fenomenólogo puede ser encontrado en la ponencia leida por Nel Rodriguez Rial en el farnoso Congreso de Fenomenologia de Sevilla (cuya traducción al inglés - Ortega Phenomenologist - se encuentra publicada en el volumen de Analecta Hussertiona que recoge las actas del mismo). Por su parte, la obra de Rodríguez Huescar $L a$ innowación metafisica de Onega (Madrid, Ministerio de Cultura, 1982) puede ser un ejemplo de lo que seria una minusvaloración de los textos más claramente fenomenologicos de dicho autor en el contexto de una interpretación general de su pensamiento.

11. La adscripción de Zubiti a la Escuela de Madrid ha resultado siempre problemática. Su contacto real con la misma puede -en efecto- no resultar suficiente como para considerarlo sin más como uno de sus integrantes, aunque su vinculacion a Ortega - quien claramente ha de ser comprendido como una de sus influencias- podria justificar tal inserción (sobre ello, vease -por ejemplo- la obra de Alain Guy. Histoire de la philosophie espagnole, Toulouse, Association des Publications de l'Université de Toulouse - Le Mirail, 1985). El parecer de J.L. Abellan y Julián Marías a este respecto podría sevir como ejemplo de aquellos que están dispuestos a defender su necesaria inclusión en dicha escuela. Por otra parte, debe señalarse la propia dificultad que puede suponer el querer definir la misma filosolia de Zubiri, pues ésta ha sido tomada como pensamiento autónomo, como ejemplo de neo-escolasticismo e, incluso, como una filosofía en mayor o menor medida fenomenologica.

12. A este respecto, nos gustaria mencionar aquí la obra de A. Ferraz Fayos Zuboni: el realismo radi cal (Madrid, Cincel, 1988), pues la misma - sin presentar una interpretación fenomenológica del pensamiento de Zubiri ni buscar una espectal vinculación del mistro con la fenomenologiacontiene una explicita mención a la cinspiración fenomenologicas (p. 26) a la que acabamos de hacer referencia.

13. Sirva como simple ejemplo la traducción de la Feromenologia de in voluztad de Pländer debida a Morente y publicada en Madrid el año 1931 por Revista de Occidente.

14. Dentro de la obra ahorn referida (que recoge las lecciones dictadas en un curso dado entre noviembre de 1960 y febrero de 1961) podría llamarnos la atención el hecho de que Gaos era perfecta- mente consciente de que la comprensión de la fenomenología hussenliana dependía de un seguimiento de la evolución del pensamiento del propio Husserl, to cual se trasluce en el hecho de que su exposición del mismo se centrara en las Meditacio nes Cartesionas, que fl mismo habia traducido. Por su parte, consideramos especialmente acertadas las afirnaciones allí vertidas sobre la profunda relacion de dependencla de la axiología scheletiana con respecto a la primitiva eidetica husserliana, aunque también es de resaltar que su tratamiento de la filosofía heideggeriana parece considerar a esta como algo ya plenamente desvinculado de su original inbricación en la fenomenologia husserliana. Por lo que hace a su aspecto crítico, baste con reproducir la siguiente afirmación (injusta, segun nuestro parecer): "Si el idealismo fenomenológico de Husser difiere del de los grandes sistemas postkantianos es pincipalmente por no tener la grandeza de estos (Filosofía contemporínea, Caracas, Universidad de Venezuela / Ediciones de la Biblioteca, 1962 , p. 222).

15. Vease su obra Dos exclusivas del hombre: la mano y el tiempo, México, 1944.

16. Permítasenos añadir que, desde una perspectiva fenomenológica, la yuxtaposición de ambos elementos no debe ser tenida $\multimap$, al menos, asi lo creemos como un violentar la fenomenología. La caracterización del hombre como faber está en Heidegger y su consideración de los entes como entespara o en su comprensión de la naturaleza de los mismos como un estara-mano e, igualmente, en Husserl y -por dar un ejemplo concreto-en el Husserl de $E l$ origen de la geometria. Piensese si no (se esté de acuerdo con él o no) en qué marca la simple posibilidad de que un trabajo como el de Tran-Duc-Tao (Phénoménologie et materialistne diaiectique, Faris, Mihn Tan, 1951) pudiera haber esperado encontrar algún eco entre alguien más que los abiertamente marxistas a, si se quiere, en el Ricoeur de Historia y verdad (vease para ello History and Truth, trad. de Charles Kelbley, Evanston, $\mathbf{I L}$. Northwestern University Press, 1965, p. 82).

17. Cerrar nuestro recorrido por la Escuela de Madrid no podría ser hecho sin una breve mención a Joaquin Xirau y en especial $-\mathrm{y}$ dejando a un lado otras cosas-a su obra La filosofía de Husserl (Buenos Aires, Losada, 1941, reeditada en 1966 por la también bonaerense editorial Troquel añadiéndole una bibliografia y un apéndice -Algunos temas del altimo Husser debidos a C.L. Ceriotto), verdadero trabajo de divulgación de la fenomenología en el que no pocos (entre los que sin rubor nos contamos) han bebido. Naturalmente, hoy no pucde hablarse del mismo sino como algo superado e insuficiente, pero no puede olvidarse que fue publicado tres años después de la muerte de Husserl y que presentaba la visión del pensamiento del mismo que a la vista de lo hasta entonces publicado 
podia tenerse. Justamente por ello (porque es un importante documento hislórico, necesario para entender qué comprensión de la fenomenologia podia tenerse por necesidad en aquel entonces) dicha obra debería ser todo menos despreciada y -aún más- habría de ser recomendada como hito necesario a quien quisiera hacerse con una panorámica de las interpretaciones de la fenomenologia con el correr del tiempo. Igualnente, tampoco podemos contentamos con terminar nuestra exposición de la recepción y divulgación de la fenomenología debida a dicha escuela sin hacer mención a la figura de R. Ledesma. Desde luego, pucde cxtrañar que ahora nos refiramos a el. pero si hemos de ser fllósofos y no apartar a Marx del ambito del pensamiento por lo sucedido tras la caida del mumo de Berlín ni condenar a Heidegger al ostracismo tras lo dicho por Farias es necesario ponerlo como ejemplo del resultado filosofico $(\mathrm{y}$, en concreto, en lo que a la recepción de la fenomenología se refiere) de la labor de los miembros de dicha escuela. R. Ledesma estudió en la Universidad de Madrid en los años álgidos del magisterio de Ortega y acudió a sus clases, licenciándose en filosofía con el tiempo justo de comenzar una incipiente labor - netamente filosofica- que truncaria su dedicación a la politica a partir de 1931. Fruto de aquel tiempo fueron una serie de artículos (todos ellos de buena factura) entre los que ahora hay que señalar los siguientes como muestra de que la primigenia introducción de la fenomenología en España fue todo rnenos algo que quedara en las alturas de la institución académica: De Rickent a la fenomonologta, Notos sobre Heidegger: ¿Qute es metafisica?, Esque. mas de Nicolai Hastmann y La ulltima incógnita de $M . S c h e l e r$ (todos ellos se encuentran reeditados en La flosofla como disciplina imperial, Madrid, Tecnos, 1983). En concreto, merece la pena recalcar el primero de los mismos, ya que -es nuestra opinion- muestra un buen conocimiento de la obra de Husserl; conocimiento capaz de llevar a su autor a enhebrar observaciones que nos recuerdan por su espiritu a algunas de las que verteria Th.W. Adorno (tan políticamente opuesto a fl) en su Zur Metakritik der Ertcentnistheorie (ahora recogida en Gesammelte Schriften, vol.5, Francfort, Suhrkamp Verlag 1970) y que chentan con el mérito de ser concisas y no tan arecargadas como las propias de la prosa de Adomo.

18. Tómense como ejemplos su trabajo «El sujeto trascendental en Husserls, en Anales del Seminario de Metafisica (Universidad Complutense de Madrid, 1966, 7-27) y su obra Estructura del conocer humano, $3^{*}$ ed, Madrid, G. del Toro, 1985 (primera edición en 1969).

19. En cuanto a obras divulgativas de J. San Martín, cabe mencionar su Estrsctura del método fenomenologica (UNED) y la publicada por Anthropos -ampliamente conocida- La fenontenologia de Hussert como ztopia de la razon. Por Io que hace a J.L. Arce (y en espera de que finalice la redacción de una obra centrada en la fenomenología), remitimos al lector a su Hombre, contocimiento y sociedad (Barcelona, PPU, 1988) donde trata la figura de Merleau-Ponty. Cabe mencionar que el magisterio fenomenológico del doctor Rábade ha llegado - Como antaño el de la primera Escuela de Madrid- hasta Hispanoamérica a través de estudiantes como R. Velozo y A. Bonilia, quienes realizaron bajo su dirección sus tesis doctorales sobre temas fenomenologicos.

20. Entre los múltiples trabajos de dicho autor dedicados a la fenomenologia pueden entresacarsc los siguientes: El problema del ente ideal (Madrid, CSIC, 1948), La estructura de la stibjetividad (Madrid, Rialp, 1967) y «La teleología del mundo físico y el nexo Brentano-Husserl\%, Revista de Filosofia (Madrid), 2/2 (1979), pp. 121-138.

21. Suya es, por ejemplo, una excelente traducción del segundo tomo de Husserliana (La idea de la fenomenologia, Madrid, FCE, 1982) y la ya referida de la quinta Meditación cartesiana, así como la revisión de la traducción original de las cuatro primeras debida a Gaos (véase la nota 6 ).

22. Husserl y la crisis de la razón, Madrid, Cincel, 1986.

23. De entre los múltiples escritos del profesor Montero dedicados a la fenomenología remitimos al lector a su Retorno a la fenomenologia (Barcelona, Anthropos, 1987). Por lo que se refiexe a Jesús Conill. puede consultarse su abra El crepilsculo de ta metaflsica (Barcelona, Anthropos, 1988).

24. La intención comunicatva, Sevilla, Themata (serie Mayor), 1989. De su labor de traducción puede encontrarse una muestra en el n." 4 de Themata (Sevilla), 1987.

25. Una de las ultimas muestras de ello serfa la obra de R.S. Ortiz de Urbina La fenomenologia de la verdad: Husseri (Gijón, Pentalfa Eds., 1984), prologada por el propio $G$, Bueno. Dentra del capítulo de autores clasificables como matcrialistas a los que ha interesado la fenomenologia cabe también señalar a L. Martín Santos, del cual se han publicado algunos fragmentos de su tesis doctoral (dedicada a Husserl y defendida en 1968 en la Universidad Complutense) bajo el título de El zigzag husserliano (Madrid, Eds. Endymion, 1990).

26. El Aprion del Mundo de la Vida. Fundamerfación fenomenologica de una ética de la ciencia y de la tecnica, Barcelona, Anthropos, 1989.

27. A este respecto cabe mencionar el trabajo ya comenzado en colaboración por J. San Martín $\rightarrow$ quien ya ha presentado traducciones de fragmentos husserlianos como apéndices a sus obras (véase nota 19)-y C. Moreno, que habrá de dar a conocer el texto de la importante Grundprobleme, Vorlesung de 1910-1911 hasta ahora ignorada por la comunidad filosofica nacional. 